\title{
Visualization Research on Scientific and Technological Information of Aviation Industry in Shaanxi Province
}

\author{
Peng Wang ${ }^{1, *}$ \\ ${ }^{1}$ Department of Photography and animation, School of art and design, Xi'an University of Technology, China
}

\begin{abstract}
The integration of industry and information is necessary for the development of industrial modernization in China. Under the context of this, the research on visualization has leaded to a wide and further integration between science and technology and arts disciplines. It fosters the progress and innovation of digitalization and smart technologies, working to make industrial manufacturing more informationalized. Besides, it has a long-term gaining effect on the digitalization and informatization of relevant national science and technology projects under way and enterprises, and even the development of education, Thus, this paper, based on the progress of aviation industry, combines visual simulation design, visual management system and visual technologies, analyses main methods and key objectives, and elaborates on the effect and significance of integration between visual technologies and manufacturing. Fund program: Special Scientific Research Program of Education Department of Shaanxi (20JK0240)
\end{abstract}

\section{Introduction}

As a pillar of the economy in China, aviation industry is foundation for the country to independently establish a solid national defense. Meanwhile, it is also the cornerstone to measure comprehensive national strength and scientific and technological strength, as well as to enhance the influence nationally and socially. In recent years, China's economic development has entered a new normal. In 2015, the State Council mapped out the development strategy of Made in China 2025 with great emphasis on intelligent manufacturing, aiming to make research and development, production, management and service more intelligent. The strategy aims to upgrade the manufacturing, promote the expanded use of information technology and industrialization, and develop a new type of manufacturing system. As the industrial development has gradually entered the crucial phase of reform and development, and transformation and upgrade, measures taken in this respect will greatly facilitate the restructuring and growth of economy.

\section{Overview}

As the top province in technological resources of aviation industry, Shaanxi gains a comprehensive strength in the proportion, research and development, manufacturing, test flight, complete sets of equipment. This paper, based on the development of aviation industry in Shaanxi province, carries out research on the application of information visualization into industrial technology in a bid to transform information and data into simulation animation. The traditional contents characterized by text and graphs will be represented more visually to consolidate the transmission efficiency and display effect of information. Specifically, centering on the key manufacturing field and crucial link of research and development, the paper is based on engineering requirements of intelligent manufacturing that integrates new-generation information technology and manufacturing equipment. And it studies how to apply the information visualization into technology. All of these efforts are made to create a more visual, quicker and clearer transmission, strengthen the communication and aid data analysis.

The extensive application of information technologies in industrialization has made the manufacturing mode more digital, automatic and intelligent, fostering the manufacturing development and working to develop China's manufacturing first. Through the integration of industrial information and visualization, emphasis is laid on the research of integrating different information layers with visualization, especially on the analysis of visual simulation design, visual management system and visual technologies. It is hoped that the modern information technologies, such as integrated techniques of digital image and 3D simulation animation, will provide more support for the growth of a digitalized industry.

\section{Visual Simulation Design}

The visual feature in 3D visualization allows some specific data in work to be displayed in a threedimensional way and improve the effectiveness of work. Besides, such technique will not only help increase work efficiency, but also further ensure work quality ${ }^{[1]}$. The

* Corresponding author: 304061024@qq.com 
simulation animation is used on the experimental data and effectiveness to visually and quickly show the experimental process and results. Moreover, through the analysis of results from visual simulation, we read data, simulate animation and choose the optimal plan, which helps us timely find the drawbacks in the model and make the plan more feasible. In brief, visual simulation design offers the important assistance in the production, assembly and experiment of aviation industry.

\subsection{Model simulation}

Visual design starts with model constructing. Specifically, simulation animation is employed for geometric modelling of buildings, workshop structure and facility and equipment. By this way, manufacturing facilities and device shapes, together with the relations of techniques are clearly and precisely demonstrated. Usually model constructing includes two methods: transforming the model with digital one or creating it according to real-life substances. As is shown in Figure 1.

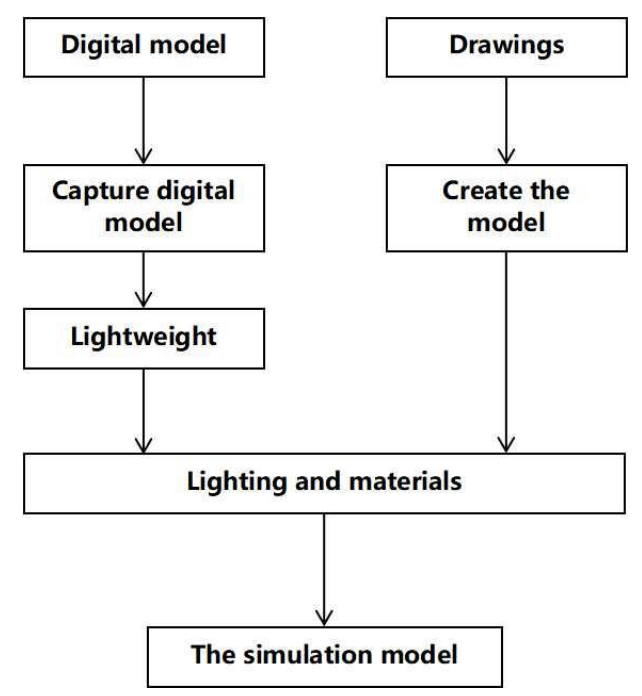

Fig. 1. Simulation model creation process.

Firstly, as for the transfer of digital model, software like MakeReal 3D can be used to capture digital model and get the simulation model. However, the model created by such approach is usually accompanied by a large volume. What's more, the model used for aviation industry is professional and precise, highlighting the practical value compared to the simulation model that stresses vision and representation. On the basis of data, this model guides manufacturing and assembling process, supporting the whole production link. Thus, the preciseness of it is higher than that of the normal one. For example, a simple package part can have thousands of polygons, and the model involving precise instrument and high-tech equipment has millions of polygons, and a complete set of production and assembly can even generate thousands of millions of polygons. From the perspective of visual representation, such a large number of polygons leads to a challenge that only in the high level of rendering condition can the rendering of model be completed. In addition, the cost is increased while the work efficiency of visualization is reduced. Therefore, it is necessary to simplify the model. Usually the command of reducing polygons in $3 \mathrm{D}$ MAX is used to proportionally lower the subdivision levels of captured model. And without affecting the model, such method can help sharply reduce the number of polygons, and the hardware requirements in the post production and rendering process.

Secondly, in terms of building simulation models, relevant graphs of production devices and environment are adopted to create models manually. Usually they are completed via computer software such as 3D MAX and MAYA, ranging from assembling package parts, designing shapes and structures, producing texture, maps, lighting and rendering based on real-life environment to the last step--building the simulation model. Meanwhile, in light of the simulation effect, the models are modified in a light weight way, characterized by hiding or deleting the model structure off camera to reduce the pressure of production and rendering. Besides, we use editing to minimize the pressure of the production without damaging the correctness of shape and structure.

\subsection{Data simulation}

After analyzing the results from visual simulation, we read data, draw curves, simulate animation and choose the optimal plan. During this process we can timely find the drawbacks of model and improve it to make the plan more feasible and scientific, which is also the core of visualization technology ${ }^{[2]}$.

Unlike the structure model, there is no specific shape or detailed process flow in the data and effect. The experimental process and results are presented by way of texts or graphs, such as stress deformation, power transmission and pressure test. Thus, while visualizing these contents, we need to consider both the visual effects and correctness of data. Additionally, data effectiveness uses analogue simulation instead of charts or texts to completely show the experimental progress or results. During the process, it should be put priority that we have a certain understanding of disciplines and properly communicate information. Then, in terms of representing the abstract contents, it is recommenced to employ post-production technologies in such software as After Effects and Premiere. Typical techniques include showing stress deformation with cloud pictures, marking values of pressure test and dynamically distinguishing area colors, all of which can further enrich the representations based on the visual simulation animation. On top of that, the visual information helps enhance the correctness of data transfer and effective information, quickly convey the industrial and technological contents, and visually present scientific and technological information. 


\section{Visual management and control system}

The comprehensive management system via visualization is crucial in the modern command and monitoring center, serving as a "bridge" between information and display. Thus, its functions directly affect the linkage of the whole system and presentation as well ${ }^{[3]}$.

\subsection{System architecture}

The manufacturing and assembling package of large and medium aircraft contain complex workflow and production information. Therefore, the visual management system is employed to build an enterpriselevel visual system, and create intelligent and informationalized management so as to achieve the integral control of links in the production chain and enhance the working efficiency. The system applies three-layer structural design, namely, information acquisition and interconnection, task decomposition and dispatch, and real-time data feedback. With the intention of overall dispatch and control, the visual transformation of integral monitoring and dynamic adjustment is achieved to obtain the dynamic dispatch, management and control in the production field. As is shown in Figure 2.

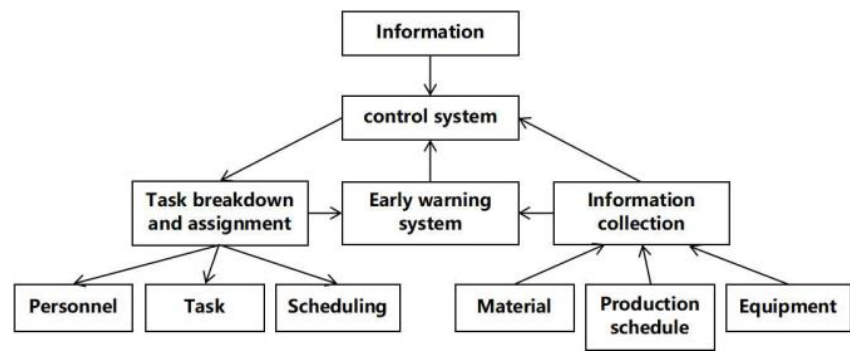

Fig. 2. Visual Management and Control System.

\subsection{System performance}

The management and control system is used to show how modules are built, and help manage the data, personnel and device during the operation process in an all-round and visual way. After receiving the manufacturing order and data given by the upper information system, the management and control system collects and associates the information and resources in the assembly field. Then, the system coordinates efforts to plan rhythm of production in line with the practical conditions of the environment, assigns work to all stations for the unified production pace, and realization of integral plan and management in the manufacturing.

To be specific, the visual system is designed for task dispatch, work assignment, assembly management, material management, tool management, device management and personnel management. Also, it centralizes on the unified scheduling of production run and shows how a system plan is given, such as date of commence, material and the release of production orders on department's command. The management system allows enterprises to know the manufacturing and inventory anytime so that they can properly manage the material and effectively control the production cost. At the same time, the system helps arrange the manufacturing schedule in the workshop. For example, it arranges the time, place and type of operation, assigns the production number, and determines the date when all phases of production are completed. After that, it is convenient to carry out the scheduling operation in the workshop and control the production records. And this can bring a scheduling report with a balanced and smoothing production so as to achieve the intelligent management of production schedule.

\subsection{System warning}

Apart from these, the computer network is employed to collect the real-time data of objectives in telemetry, tracking and command. Meanwhile, we use remote control to provide necessary and complete data and means for production monitoring, safety, optimization, and trouble shooting. In accordance with the practical management requirements, various data warnings, which include monitoring system, pre-warning information transfer, pre-warning system and forecast evaluation, are achieved in many aspects, such as production schedule, inventory warning and test pre-warning. To obtain the relevant information and operation state, we perform a real-time analysis of data detection in the main links of production, and then evaluate the production process according to the integrated information and data. In the final delivery, we do a shakedown test on the machine to rapidly monitor the production quality and timely adjust the rhythm of production.

\section{Application of visualization technology}

By visualizing scientific and technological information, we transform information and data into simulation animation, representing the traditional textual and graphic contents in a more visual way. This method will definitely enhance the display effect of information, guide the project construction and management, and finally facilitate the integration and management of industry and information.

\subsection{Visual display}

In the virtual and visual environment, the manufacturing capacity and process, along with all aspects involved such as production devices and layout of workshop can be simulated. Through this way, people will know and analyze the manufacturing process of products before the actual processing[4]. The visual management system and simulation model is combined to reproduce the enterprise buildings, working structures and facilities and devices so that the features of devices and equipment, and relations of manufacturing techniques are visually and precisely represented. In other words, 
the visual demonstration helps gather the information which exists in every link of manufacturing in a model, and then display it. By this way, the different views can help examine the overall production environment and process, facilitating the examination and monitoring of production environment. To sum up, this application helps build a management platform for dynamic devices in the "intelligent factories", whose purpose is to make industrial manufacturing more digital, automatic and intelligent.

\subsection{Simulation training system}

As the people who have the most access to devices, operators are quite familiar with various operation features and regularities of devices, and thus are crucial as for whether devices are operated well[5].Nowadays, as the introduction of visualization changes the way training information is transferred, the graphic representations enable operators to improve their skills. Specifically, it is available for them to understand working process and regularities visually, conveniently and efficiently. During the transformation of textual information to images and graphs, they summarize and conclude effective information, giving a full play to the efficiency advantage of images in the information representation. Operators extract information through conceiving the visual images, which can help them quickly understand and master working techniques and rules. For example, the visual demonstration is utilized to create simulation animation for complex or specific working procedures, make explosion models for equipment and devices, and provide graphs and images. All of these assist workers in rapidly finding a solution in production operation, device maintenance and troubleshooting.

Combining visual representation, we can establish systematic training plans for different modules such as enterprise environment, device operation, production process and management system. Meanwhile, it is visualization that helps interpret the information and hence has a strong practical significance on the personnel training and company development. As is shown in Figure 3.

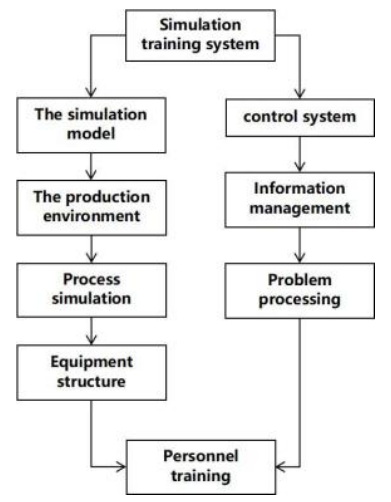

Fig. 3. Simulation training system.

\section{Conclusion}

The integration of industrial production and visualization has marched towards the manufacturing industry, and is particularly well-accepted in the application of aircraft industry. Such integration involves virtual reality, artificial intelligence and economic category, keeping close contact with the advanced science and technology. In particular, visualization is applied in production, assembly and experiment, which lays foundation on the enterprises to modernize. Nowadays, China is shifting from a big manufacturing country to a strong one for the transformation of manufacturing. As visualization is essential in the scientific and technological environment, it is suggested to proactively conduct research on it, promote its integration with other disinclines to take full advantage of it. The research on visualization and intelligence shows the way that medium and large assembly and manufacturing industries develop. It is believed that visualization will have a brighter future soon and contribute to the social progress and development.

\section{References}

1. J. X. Lv, C. F. Wang, W. Han, G. Wu, Intelligent computer room application based on 3D visualization technology[J], Electronic Technology \& Software Engineering, 2019

2. S. Wang, Application of visualization technology in intelligent manufacturing[J], Electronic Technology \& Software Engineering, 2017

3. . Dong, J. S. Li, S. N. Wu, Application of Visual Integrated Mangement and Control System[J], Intelligent Building, 2019

4. R. X. Ning, J. H. Liu, C. T. Tang, Modeling and Simulation Technology in Digital Manufacturing[J], Chinese Journal of Mechanical Engineering, 2006

5. H. Qiao. Y. Liu, B. You. X. Zheng, Design and Implement of the Visual Simulation Training System for Boilers in Industrial Enterprises[J], Journal of Chengdu Technological University, 2019

6. H. R. Zhao, J. H. Liu, H. Xiong, C. B. Zhuang, T. Miao, J. S. Liu, B. Wang, 3D Visualization RealTime Monitoring Method For Digital Twin Workshops[J], Computer Integrated Manufacturing Systems, 2019

7. Q. Z. Pan, X. W. Meng, R. C. Fei, Data visualization technology and its application prospect[J], Electronic Technology \& Software Engineering, 2017 